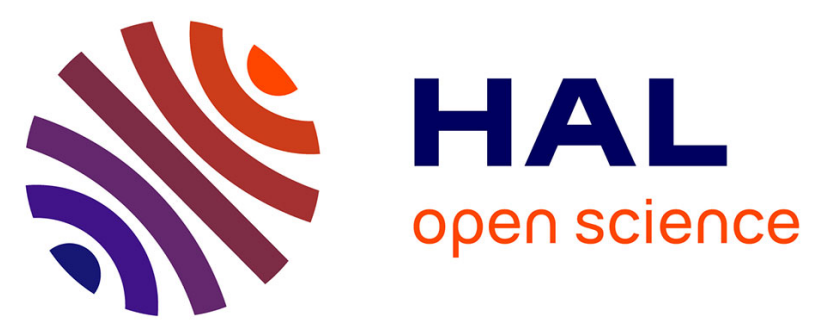

\title{
Which models are used in social simulation to generate social networks? A review of 17 years of publications in JASSS
}

Fredéric Amblard, Audren Bouadjio Boulic, Carlos Sureda Gutierrez, Benoit Gaudou

\section{- To cite this version:}

Fredéric Amblard, Audren Bouadjio Boulic, Carlos Sureda Gutierrez, Benoit Gaudou. Which models are used in social simulation to generate social networks? A review of 17 years of publications in JASSS. Winter Simulation Conference (WSC 2015), Dec 2015, Huntington Beach, CA, United States. pp. 4021-4032. hal-01303799

\section{HAL Id: hal-01303799 \\ https://hal.science/hal-01303799}

Submitted on 18 Apr 2016

HAL is a multi-disciplinary open access archive for the deposit and dissemination of scientific research documents, whether they are published or not. The documents may come from teaching and research institutions in France or abroad, or from public or private research centers.
L'archive ouverte pluridisciplinaire HAL, est destinée au dépôt et à la diffusion de documents scientifiques de niveau recherche, publiés ou non, émanant des établissements d'enseignement et de recherche français ou étrangers, des laboratoires publics ou privés. 


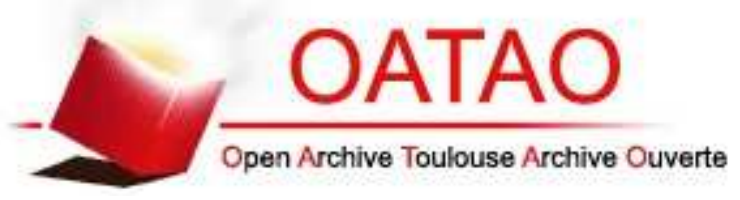

\section{Open Archive TOULOUSE Archive Ouverte (OATAO)}

OATAO is an open access repository that collects the work of Toulouse researchers and makes it freely available over the web where possible.

This is an author-deposited version published in : http://oatao.univ-toulouse.fr/ Eprints ID : 15490

The contribution was presented at : http://www.wintersim.org/2015/

To cite this version : Amblard, Fréderic and Bouadjio Boulic, Audren and Sureda Gutierrez, Carlos and Gaudou, Benoit Which models are used in social simulation to generate social networks? A review of 17 years of publications in JASSS. (2015) In: Winter Simulation Conference (WSC 2015), 6 December 2015 - 9 December 2015 (Huntington Beach, CA, United States).

Any correspondence concerning this service should be sent to the repository administrator: staff-oatao@listes-diff.inp-toulouse.fr 


\title{
WHICH MODELS ARE USED IN SOCIAL SIMULATION TO GENERATE SOCIAL NETWORKS? A REVIEW OF 17 YEARS OF PUBLICATIONS IN JASSS
}

\author{
Frédéric Amblard \\ Audren Bouadjio-Boulic \\ Carlos Sureda Gutiérrez \\ Benoit Gaudou \\ UMR 5505 CNRS, IRIT \\ University Toulouse 1 Capitole \\ 2 Rue du Doyen-Gabriel-Marty \\ Toulouse, FRANCE
}

\begin{abstract}
Aiming at producing more realistic and informed agent-based simulations of social systems, one often need to build realistic synthetic populations. Apart of this synthetic population generation, the question of generating realistic social networks is an important phase. We examined the articles published in the Journal of Artificial Societies and Social Simulation (JASSS) in between 1998 and 2015 in order to identify the models of social networks that were actually used by the community. After presenting the main models (regular networks, random graphs, small-world networks, scale-free networks, spatial networks), we discuss the evolution of the use of each one of these models. We then present different existing alternatives to those kind of models and discuss the combined use of both simple and more elaborated or data-driven models to different aims along the process of developing agent-based social simulation with realistic synthetic populations.
\end{abstract}

\section{INTRODUCTION}

When wanting to develop agent-based social simulation of a phenomenon on a specific area (let's take as an example a model of H1N1 propagation in the region of Danang, Vietnam), modellers face the problem of synthetic population generation. Aiming at populating their models with realistic individuals, they often have access to global statistics (age pyramid, income distribution...) they have to disaggregate in order to generate a realistic synthetic population of agents on their case study. Dealing with individual attributes (age, sex, income...) the problem is tractable; it is more or less the case of their location when they want to include a spatial dimension to their model. But in the case of social networks generation they face a dilemma, the data are usually not available or they are very limited and the most known models (Small-world networks, scale-free networks for instance) seems far too abstract to capture the corresponding social structure on their case study.

Before entering into the presentation of the existing possibilities for synthetic social networks generation from the literature, let us summarize the issue faced by the modeller when wanting to generate such a network. In order to present it simply, the generation of social network may take place hereafter the generation of the synthetic population and eventually its geographical localization in an environment. Therefore we can consider that before entering this final step we have a population of agents that possess attributes (age, sex, income, home location, work location ...) and we want to interconnect them through a social network that will model the possible interactions taking place among the individuals. The social 
network component is in particular useful when modelling epidemic spreading (Eubank et al. 2006), opinion dynamics (Amblard and Deffuant 2004) or economic transactions (Tesfatsion 2002) in a population.

The social network we want to generate at the initialization of the simulation is therefore a static graph composed of nodes (representing the individuals/agents) and links among them. The nodes may be described by different attributes (as the ones generated from the preceding step) as it may be the case of the links. Concerning the main properties that could be associated to links, we generally find the following ones:

- links may be oriented or not (I lent you money or we work together)

- links may be weighted or not (how much I lent you)

- links may be labelled or not

- several kinds of links may be considered (multiplex networks) or not.

These different options strongly depend on the model and modeller's approach of the issue. As concerning the static or dynamic property of this graph we placed a clear limit in between the generation of the initial state of the model (that is clearly our scope) and the modelling part (for instance the possible evolution of the social network, that corresponds to hypotheses taken by the modeller and are clearly, at least for the moment, out of the scope of our approach). Even if the generation of this initial static graph may be the result of a dynamic process modelling social network evolution (as it is the case for instance of the Preferential Attachment model (Barabasi and Albert 2001)), we are, in this case, only interested in the final output of this dynamics and not for instance in the psycho-sociological soundness of the process that enables such a generation. Moreover, and although it is a very promising perspective, we won't mention multiplex networks and will only consider they can be generated by the repetition of some of the listed approach applied independently on the different layers, even if it is a strong assumption and if we anticipate some co-dependencies in between the layers of a multiplex network.

Therefore dealing with the generation of this initial static graph, one can distinguish in between two main features that enable to generate the network as well as two different scales for this generation. Concerning the generation of the network, we can basically take two kind of information: the attributes or profiles of the nodes (making the creation of links depends on the profiles of the two concerned agents following for instance an homophilic process) and the structural properties of those nodes (considered in general by dynamical generation processes, when searching for instance to mate individuals that have similar number of links). The two different scales considered enable also to categorize the solutions. Basically we can distinguish in between local processes where agents apply rules to their local neighbourhood in order to generate or not corresponding links, and global or macroscopic approaches where one consider the graph as a whole and apply link creation rules from a top-down approach.

In order to clarify and structure our presentation of the state of the art, we will first focus on a representative journal for the community of social simulation, namely the Journal of Artificial Societies and Social Simulation (JASSS). From this journal we made a systematic review of the models used to generate social networks in the different models. We will present this study in the section 2 and at the same time the models used by the community. We will end the section 2 by discussing the evolution of models used for the generation of social networks in this field along its 17 years of existence. In the section 3 we will extend qualitatively our study presenting other kinds of social networks generations published outside of JASSS to broaden the scope. Finally we will present other approaches for which we didn't find yet any related agent-based model but for which we see an interesting potential.

\section{DATA COLLECTED ON JASSS}

Searching for a representative dataset of the practices at play in the social simulation community, we decided to focus in the Journal of Artificial Societies and Social Simulation (JASSS) which exists since 1998, created by Nigel Gilbert and is one of the main journal of the social simulation community (other journals are for instance Computational and Mathematical Organization Theory, Advances in Complex 
Systems, Complexity). The two main interests to choose this journal is on the one hand it is in open access and on the other hand it explicitly focus on social simulation whereas other journals could have a broader scope on complex systems in general. From its creation in 1998 to its last issue in March 2015, JASSS published 628 articles. Among these 628 articles that we browse one by one we selected the papers that were mentioning explicitly the terms "social network" in the description of an original model (then excluding surveys as well as methodological papers). Then searching for these papers the methods used to generate the initial social network, we excluded again: papers concerning the modelling and simulation of network dynamics for which the initial state is either an empty graph or a full graph (making evolve, as it is the case for several papers, the weights of the links depending on agents' behaviours) ; papers concerning supply-chain simulation, although they can be considered more or less as a social network in-between agents, they composed a quite specific family, and the generated networks are trivial (linear ones) and rarely includes more than a decade of agents ; papers concerning infrastructure networks (either roads, or communication channels) ; moreover we also excluded 4 other papers because of the lack of specification concerning the social network models they used.

At the end of this filtering, we finally kept in our dataset 93 papers, published in between 1998 and 2015. From this dataset, we classified the set of models used and their representativeness from the dataset (cf. Fig.1).

We sorted the different models referenced into 9 categories:

- Regular lattices (27\%) inherited quite naturally from the cellular automata approach and regroup mainly 2D-lattices with either Von Neumann or Moore neighbourhood, but also (even if it is a minority) 1D-lattices.

- Random networks (29\%) correspond mainly to the Erdös-Rényi model.

- Small-world networks (21\%) correspond in fact to the $\beta$-model (Watts 1998).

- Scale-free networks (19\%) correspond mainly to the Preferential Attachment model of (Barabasi and Albert 2001) but not exclusively.

- Spatial networks (20\%) correspond to networks built from spatial distribution of the agents using for instance a distance below which agents are either systematically or stochastically connected.

- Hierarchical structures (5\%) correspond to tree-like graphs and are used in general either to generate organizational structures or familial networks

- Kinship networks (5\%) correspond to the generation of networks modelling familial networks and using in general bi-partite graphs to distinguish in between males and females.

- Empirical networks (3\%) correspond to cases where empirical data on social networks are used to generated the social network, where the empirical data that are used do not necessarily concerns the target system to be modelled but may concerns another system.

- Other kind of models (13\%) regroup mainly ad hoc models of social networks strongly related to the population being modelled and also very ideal networks (star-network, linear ones), these latter being a minority for this category. 


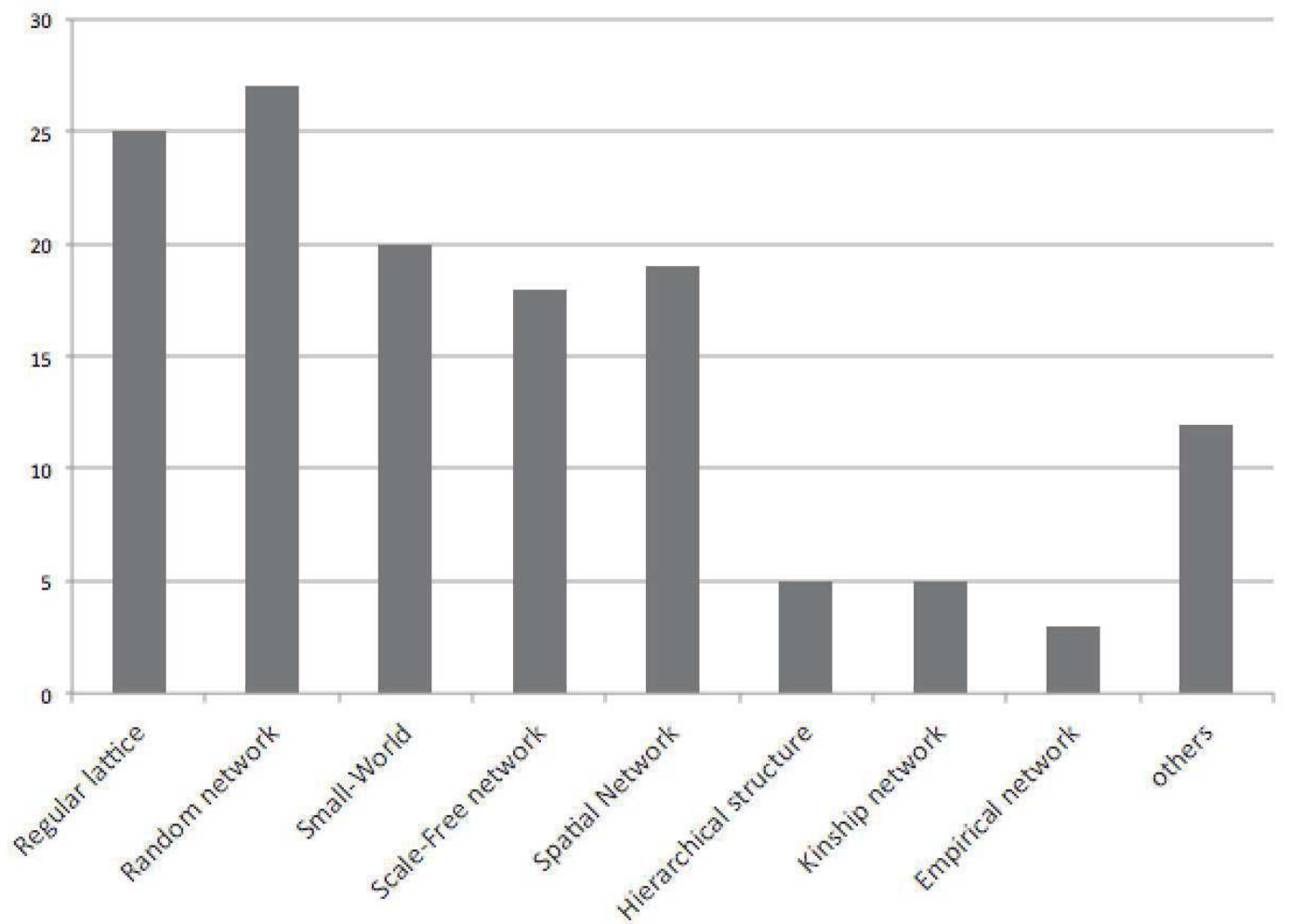

Figure 1: Number of JASSS articles where the related models are used in between 1998 and 2015.

The scrupulous reader has yet noticed that the total of the percentages are actually far upon $100 \%$, it is due to the fact that in certain papers, several networks models are used in order for instance to test the influence of the kind of network on the results of the simulation (for instance, for several papers, testing an agent-based simulation on random networks, small-worlds and scale-free networks (Suo and Chen 2008)).

We will now present into more details the different models in question and then the evolution of the use along the 17 years of JASSS the first four being presented in chronological order.

\section{MODELS USED IN AGENT-BASED SOCIAL SIMULATION FROM THE JASSS SURVEY}

\subsection{Regular networks}

The field of social simulation inherited of many tools coming from the complex systems field and in particular of cellular automata (Wolfram 1986). The corresponding underlying interaction structure is then in general the one of a regular grid and in many cases a torus. The agents are then represented by the cells of the automata and their social neighbourhood is defined from the regular grid with a von Neumann's or a Moore's neighbourhood. The Newman's neighbourhood links a cell to its four neighbours (North, East, South, West) as Moore's neighbourhood adds four more neighbours (NE, SE, SW, NW) and extended Moore neighbourhood adds other cells depending from their distance (in terms of number of cells) to the central agent. A representative instance of such approach is the article of Leydesdorff (2001) concerning innovation diffusion in socio-economic networks.

However they are quite unrealistic, each agent having the exact same number of neighbours that are either 4, 8, $24 \ldots$ They have been and actually still used as they present a big advantage compared to other existing models: they enable a clear visualization of the simulated phenomenon. As for instance with 
diffusion process, one can easily visualize the spread happening spatially as there is a perfect mapping in between the social structure and the underlying space.

\subsection{Erdös Rényi random graph model}

From there, some modellers defined the interaction structure of their models by using a random graph following the model of Erdos and Renyi (1960). A random graph is a set of $N$ nodes connected by $n$ edges which are chosen randomly from the $N(N-1) / 2$ possible edges. A great advantage of this model compared to the regular graphs is that you can easily play on the average connectivity of the graph, as it was fixed to $4,8,24 \ldots \mathrm{n}$ in the former case; also, on a pragmatically side, this model is quite easy to implement.

Dealing with the features and properties of such random networks, once the population (number of nodes) is fixed, the only parameter of the model is the links density (or the average connectivity of the nodes). The links density presents a percolation threshold at $\ln N / N$. Under this critical threshold, the network is very likely to be disconnected and presents several components; above it the model exhibits a single giant component. Moreover, the model presents low clustering as well as low diameter (or a low average path length among pairs of nodes). Dealing with the nodes' degree distribution, it follows a Poisson law centred on the average connectivity. The clustering is lower than regular networks of the same size. A representative publication from our dataset using ER model is the consensus model proposed by Stocker et al. (2001).

\subsection{Small world models}

The arising question at this stage was: do some graphs between these two extremes (regular and ER networks) that may have other characteristics exist? A first class of models comes from the Watts and Strogatz's work, i.e. the class of Small World graphs (Watts and Strogatz 1998; Watts 1999). They propose algorithms that enable to fill the gap between regular structures and random graphs. The second one they proposed, the $\beta$-model, which is actually the one that is effectively used in agent-based social simulations, is based on a substrate, a starting structure that is regular. Hereafter, depending on the $\beta$ variable, between 0 and 1, it keeps or rewires at random each link of the existing graph.

It yields to the introduction on a regular structure of random graphs characteristics especially the reduction of the average path length among the agents. These Small World graphs inherit in a way of each one of the properties of both regular and random graphs. They have both a high clustering coefficient and a low averaging distance. It follows, for the scientists who test their models on this class of graphs that their models were functioning very differently (Axtell 2000; Soorapanth et al. 2001).

From these different models, the $\beta$-model is definitely the most used, as in Huang at al. (2005). The fact is that this model is very simple to implement and enables very easily to play both on average connectivity and the randomness degree of the graph. The clustering property in particular that is identified from real social networks was enabled by such model. However, a property that is not present in the generated graphs from this model concerns the degree distribution (or connectivity of nodes). In several empirical social networks a strong inequality has been shown concerning such distribution with a vast majority of the individuals having few links whereas few ones have many (Liljeros et al. (2001) for instance on sexual networks). Dealing with this property the small world graphs exhibits rather a Poisson law distribution smoother and smoother as the $\beta$ parameter grows.

\subsection{Scale-free networks}

Confronting models to empirical data on several kinds of networks, as the Internet or the metabolic network, Barabasi's team exhibits a power-law distribution of nodes' degree (Albert et al. 1999). It is a property they do not encounter in the existing theoretical models. Then they propose the model of preferential attachment (Barabasi et al. 2000) which has the degree distribution as an input, but is random 
in all other aspects. The preferential attachment model follows an iterative process. Starting from a seed of nodes and adding new ones, it adds also corresponding links following the rule that the more a node is connected the more it has chance to receive new ones. This probability is tuned by a parameter of the model. Then the model of scale-free networks can be applied in particular to generate random graphs with a degree distribution that follows a power-law.

Within this class of graphs several directions are proposed to modify it. In Albert and Barabasi (2000) they propose a rewiring edges dynamics that also provides a links dilution over time. Dorogovtsev and Mendes (2000) propose in the same way a stochastic algorithm that add edges between old sites and that can remove existing edges, the probability of link creation between two nodes being proportional to the product of the their degrees. Amaral et al. (2000) propose an aging cost or a capacity constraint to new link creation that refrain the process of power-law degree distribution.

The use of such generated networks (as in Stauffer et al. (2001)) is still subject to discussions as on the one hand, the properties of such networks are very particular compared to others and their use is therefore informative concerning the behaviour of the model. On the other hand, the features of scale-free networks and especially the strong inequalities among nodes concerning their connectivity, if they are identified in some cases of social networks (Liljeros et al. 2001) do not fit with underlying assumptions in many social systems.

\subsection{Spatial networks}

Models which are regrouped in this category are basically built from an underlying space, often geographical but sometimes interpreted as a social space (where each dimension covers an individual attribute). Agents are then located in this space and a distance threshold is applied in order to build the corresponding network linking closest agents either in a deterministic or stochastic (applying probabilities of connections) way. The social circles model (Hamill and Gilbert 2009) is a good instance of this kind of models. Dividing agent in several group with different range, we can obtain a social network with some desired properties, like strong clustering, assortativity and correct path length, as well as fat tail degree distribution, depending on the agent group's distribution. In the case of a social space, the process at play is built upon homophily among agents. Barthélémy (2011) published a survey dedicated to spatial networks where he proposed some spatial variations of the most used models, e.g. ER graph, small world, or preferential attachment. Basically, a cost is associated with the distance between the nodes to be linked, affecting the probability of the association. It will then have consequences on the network properties, increasing clustering and average shorter path, or raising the correlation between position and centrality to the one between centrality and degree, for example.

\subsection{Other kinds of models used}

In this latter group we won't detail the models proposed as they are very heterogeneous. However, some of them could be regrouped depending on the type of network they are searching to generate. In the case of organizational networks, hierarchic networks (basically trees that could integrate some probabilistic rewiring) are used intuitively (Kim 2009), whereas for the generation of kinship networks (Yang et al. 2009), the models proposed are more focused on the building of couples (random bi-partite graph in between men and women) and then the growth of those proto-familial network integrating children (linked to their parents) sometimes over several generations.

As dealing with empirical networks, none of the article published in JASSS is built upon empirical network corresponding to the modelled system (the reason behind is of course linked to the lack of availability of such precise data). However, Cointet and Roth (2007) evaluated several social network models as well as empirical networks in order to determine their capacity to enable and reproduce knowledge diffusion process. Their conclusion is surprizing and interesting as using an other empirical social network of roughly the same size is far better than using the abstract models available. Two other more recent articles published follow the same methodology successfully. 
To conclude with the social networks models used in JASSS, the last category "others" corresponds to ad hoc models of social networks that follow strongly the modelled system. However interesting, they are poorly generic and cannot really be adapted to model other systems.

\section{RESULTS OF THE JASSS CORPUS ANALYSIS}

The presentation of the different models being done, one could wonder if the usage of a particular model evolved along time in the community or if they do remain more or less constant in time. In order to obtain a clear picture of such an evolution, rather than using a yearly representation (the samples being too poor to represent correctly tendencies in time), we regrouped the articles into periods of 5 years (for the first and last segments as they correspond to less publications) and 4 years (the two other segments 2003-2006 and 2007-2010). The result (cf. Fig. 2) illustrates well the main tendencies in the field.

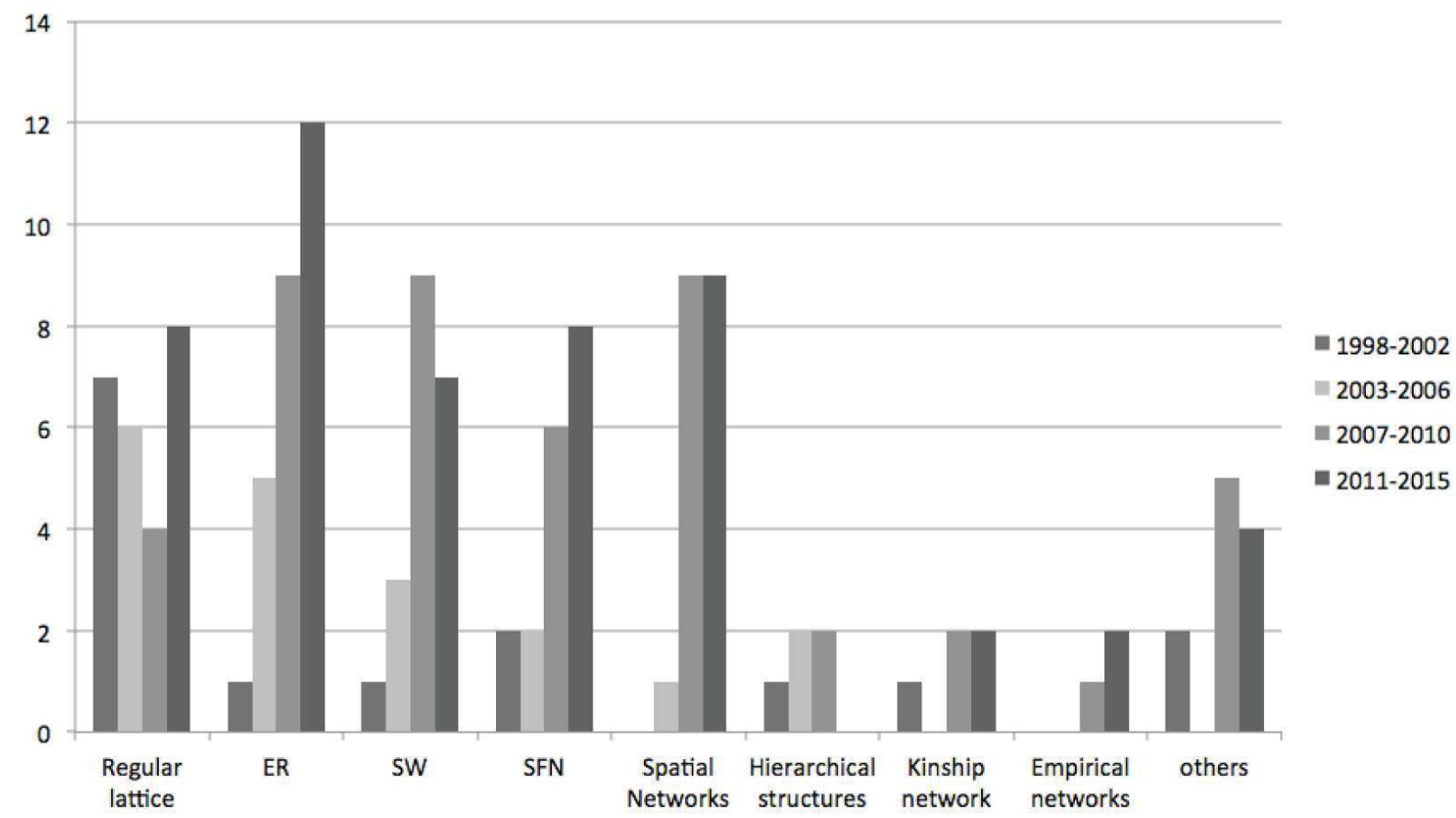

Figure 2: Number of publications including each type of networks on 4 and 5 years segments in between 1998 and 2015. (ER: Erdos and Renyi, SW: Small World, SFN: Scale Free Network).

To draw a rough picture of these evolutions in the domain of social simulation, we have to start back at the origins and especially from the cellular automaton ancestor. As agent-based simulation was originally built from this approach, first models (like the segregation model of Schelling for an instance) were built on checkerboards or regular grids. It appears from there a kind of bifurcation concerning the interpretation of this grid.

For a first category of the modellers, this grid represented a discretization of a geographical space, agents in their models being able to move on this grid, it quickly evolved into models of the environment that were no more discrete but continuous (as far as possible in computer science) and agents moving no more from square to square but rather continuously (think about the Boids model of Reynolds for instance). When, following the mood, they then wanted to integrate social networks into their models, it was quite natural to build them using distance among agents, it is probably one of the ways spatial networks have been adopted (rather than actually proposed) by the social simulation community. 
For the second category the original grid of the cellular automata was rather abstractly as an interaction structure, that they very soon called a social network. The different properties of the corresponding social structures, the models were integrat Erdös-Rényi model enabling to test easily the sensitivity of simulation outputs to t] network, Small-world enabling to test moreover the sensitivity to the randomness of eventually the clustering), scale-free networks enabling to test the sensitivity to high i connectivity among nodes.

The global picture can be probably more precise when looking at the aim of the $\mathrm{mc}$ use social networks models. If in the first periods those models were envisaged as realistic representation of the social networks (these are actually the kind of argum publications) compared to regular grids, the "social network effect", i.e. the fact that social network has an impact on the simulation outputs, conducted progressively many ( actually test such a network effect by using different models of social networks. In the is for instance very frequent to actually see the conjoint use of Erdös-Rényi, small-wo] scale-free networks to try and evaluate this network effect. Therefore as a part of sensiti models, even quite far from realistic social networks, are actually useful.

However, two problematic issues remain: on the one hand the use of such models ( synthetic representation of social networks (see again the argument of Cointet and Ro the other hand the use of ad hoc models that are difficult to reproduce, not very well : add usually to the model a bunch of new parameters.

\section{PERSPECTIVES: WHAT WOULD BE THE ALTERNATIVES?}

From this situation, three directions can be envisaged for social networks models that this part. The first one concerns further development and use of abstract models. $T$ dedicated to the generation of social networks from sample data. Finally we will pres solution to build modelled population on a network (as opposed to ad hoc network mode

\subsection{Is there a future to abstract models?}

As such the four main representative models of this category, namely regular, random scale-free networks, when used in the frame of a sensitivity analysis to test density-depe instance, are totally credible. Moreover one of the big advantages of these models is 1 easy to implement, enabling then the reproducibility of the experiments as well as a fa such tests for any modeler. Probably we should wonder whether we should wait for ye model of the same kind?

The Forest Fire model (Leskovec et al. 2007) enables to create networks having mos observed in real networks, in particular communities and skewed distribution of degree any agent-based social simulation where such model was used to generate the social net is a promising one, just as other models or benchmarks (Lancichinetti et al. 2008) tha test community effects or mixing of populations in the model. However interesting difficultly appear as convincing solution for the generation of synthetic social network social simulation.

\subsection{Sampling approaches}

Another set of approaches that can be used in this particular frame to generate synth different methods and tools enabling to do some sampling approaches. The very basic is in this case is to start from a network sample on the target system, then to find a mo statistical) describing this sample and from there generate the network needed to con population of the agent-based model. 
Exponential Random Graph Model (ERGM) (Snijders et al. 2006; Robins et al. 2007a,b) are a family of statistical models that allow to represent empirically observed networks. Each network tie is regarded as a random variable and relations between agents are considered stochastic. It is a generalisation of Markov Random Graph Model (MRGM) that takes into account some parameters like edge, triangle and $\mathrm{k}$-star count but has a degenerate nature that prevents to represent well transitivity as observed in many social network, since in almost all graphs generated by MRGM the transitivity is either quite weak or much too strong. A discussion on ERGM and an alternative, the Link Probability Model is presented in (McCulloh et al. 2010).

Among the possible solutions to find the corresponding model, we can also use Kronecker graphs (in particular stochastic Kronecker graphs) as proposed by Leskovec (2008) that enables to find a matrix pattern that you will replicate at different scales. However promising the Kronecker graphs do not enable to manage accurately the population size that should corresponds to a power of the matrix pattern size.

Menezes and Roth (2014) proposed a different approach to generate a network based on a sample. Their method aims to discover and select plausible generators, i.e. processes and rules for real-world networks through a genetic algorithm. Generators can contain operators (arithmetic, classical mathematical functions as $\log$, abs, etc., conditional expressions and an "affinity function"), variables (in- and outdegrees, several distances between two agents and sequential identifiers) and constants.

Once we do obtain a functional representation of the sample network, the generation of a more complete network connecting the synthetic population generated is then possible.

\subsection{Rule-based approach for the generation of social network}

Another alternative is envisaged by Thiriot and Kant (2008) with the corresponding software YANG (Yet Another Network Generator) (http://sourceforge.net/projects/yang-j/). The global approach consists in generating a synthetic population using Bayesian rules and from there to generate the corresponding social network by specifying the rules for the matching in between individuals (for instance agents will tend to have friendship relations with agents of the same sex and of approximately the same age). Therefore using a Bayesian network and scattered statistics, the software will generate a network under the specified constraints. Such approach even if it could be considered as a generator of ad hoc models is indeed far more robust than these latter approaches as the global process is formalized, could be reproduced and the networks generated can be easily analysed.

We have also to reference all the work achieved on the MIDAS and EPISIM projects, developed in particular by the team of Stephen Eubank, aiming at building synthetic populations and therefore synthetic social networks, in particular in the frame of epidemiological dynamics. All along their project they evaluated existing solutions for network generation and proposed very innovative ones with promising results (Eubank et al. 2004; Eubank et al. 2006).

The question of synthetic network generation is actually moving very quickly, first the increased willingness to include social networks representation in agent-based simulation as well as the increased use of data-driven approaches to obtain much realistic and reliable models are putting pressure to obtain reliable methods to generate such synthetic networks. Moreover the very strong evolution concerning availability of data on social networks introduces an important change in the problem definition and in the possible solutions to be proposed. Where 10 years ago, very few (and expensive in time and effort) data where available on social networks and especially longitudinal social networks, nowadays automatic solutions for the collect of social networks data (through either smart devices or RFID chips) enables a facilitated access to those data.

\section{CONCLUSION}

In this article we presented a survey of the methods used to generate social networks in the field of social simulation, based on the articles published in JASSS in between 1998 and 2015. From this survey it appears that the large majority of publications $(69 \%)$ were actually using very simple models (regular 
lattices, random graphs, small-world networks or scale-free networks). We analyzed the evolution of this use all along these 17 years of publications and identified two streams of evolution issued from the original cellular automaton ancestor: on the one hand the spatial approaches and on the other hand the approaches corresponding to social structure. We then presented mostly recent (to the exception of ERGM models) approaches that could be envisaged as convincing alternatives to simple models for the generation of synthetic social networks.

\section{ACKNOWLEDGMENTS}

This work benefited from the support of the project GENSTAR (ANR- 13 - MONU - 0006) of the French National Research Agency (ANR).

\section{REFERENCES}

Albert, R., H. Jeong, and A.-L. Barabási. 1999. "Diameter of the World Wide Web", Nature 401: 130131.

Albert, R., and A.-L. Barabási. 2000. "Dynamics of Complex Systems: Scaling Laws for the Period of Boolean Networks", Physical Review Letters 84: 5660-5663.

Amaral, L. A. N., A. Scala, M. Barthélémy, and H.E. Stanley. 2000. "Classes Of Small-World Networks". Applied Physical Sciences 97(21):11149- 11152.

Amblard, F., and G. Deffuant. 2004. "The Role of Network Topology on Extremism Propagation with Relative Agreement Opinion Dynamics", Physica A 343: 725-738.

Axtell, R. 2000. "Effects of Interaction Topology and Activation Regime in Several Multi-agent Systems." In Multi-agent based simulation. S.Moss and P.Davidsson (Eds.). Lectures Notes in Artificial Intelligence 1979: 33-48.

Barabási, A.L., and R. Albert. 1999. "Emergence of Scaling in Random Networks". Science, 286(5439): 509-512.

Barabási, A.-L., R. Albert, and H. Jeong. 2000. "Scale-Free Characteristics of Random Networks: the Topology of the World-Wide Web", Physica A: Statistical Mechanics and its Applications, 281(1-4): 69-77.

Barthélémy, M. 2011. "Spatial Networks", Physics Reports 499(1): 1-101.

Bodine-Baron, E., B. Hassibi, and A. Wierman. 2010. "Distance-dependent Kronecker Graphs for Modeling Social Networks", IEEE Journal of Selected Topics in Signal Processing, 4(4): 718-731.

Cointet, J.-P., and C. Roth. 2007. "How Realistic Should Knowledge Diffusion Models Be?", Journal of Artificial Societies and Social Simulation 10(3): http://jasss.soc.surrey.ac.uk/10/3/5.html.

Dorogovtsev, S.N., and J.F.F. Mendes. 2000. "Evolution of Reference Networks with Aging." Physical Review E, 62:1842.

Eubank, S., H. Guclu, and V.S.A. Kumar. 2004. "Modelling Disease Outbreaks in Realistic Urban Social Networks". Nature 429:180-184.

Eubank, S., V.S.A. Kumar, M.V. Marathe, A. Srinivasan, and N. Wang. 2006. "Structure of Social Contact Networks and their Impact on Epidemics", In Discrete Methods in Epidemiology, Abello J, Cormode, G (ed.). DIMACS Series in Discrete Mathematics and Theoretical Computer Science 70:179-185.

Erdös, P., and A. Rényi. 1959. "On Random Graphs”. Publicationes Mathematicae 6: 290-297.

Green, D.G., T.G. Leishman, and S. Sadedin. 2008. "Dual Phase Evolution-a Mechanism for SelfOrganization in Complex Systems", In Unifying Themes in Complex Systems, Springer Berlin Heidelberg: 58-65.

Hamill, L., and N. Gilbert. 2009. "Social Circles: A Simple Structure for Agent-Based Social Network Models". In Journal of Artificial Societies and Social Simulation 12(2)3 http://jasss.soc.surrey.ac.uk/12/2/3.html. 
Holland, P.W., and S. Leinhardt. 1981. "An Exponential Family of Probability Distributions for Directed Graphs", Journal of the American Statistical Association, 76(373): 33-50.

Huang, C.-Y., C.-T. Sun, and H.-C. Lin. (2005). "Influence of Local Information on Social Simulations in Small-World Network Models", Journal of Artificial Societies and Social Simulation, 8(4), http://jasss.soc.surrey.ac.uk/8/4/8.html.

Kim, W.-S. (2009). "Effects of a Trust Mechanism on Complex Adaptive Supply Network: an AgentBased Social Simulation Study", Journal of Artificial Societies and Social Simulation, 12(3), http://jasss.soc.surrey.ac.uk/12/3/4.html.

Lancichinetti, A., S. Fortunato, and F. Radicchi. 2008. "Benchmark Graphs for Testing Community Detection Algorithms". Physical Review E 78(4):046110.

Leishman, T.G., D.G. Green, and S. Driver. 2009. "Self-Organization in Simulated Social Networks". In Computer-Mediated Social Networking, Springer Berlin Heidelberg: 150-156.

Leskovec, J., J. Kleinberg, and C. Faloutsos. 2007. "Graph Evolution: Densification and Shrinking Diameters". ACM Transactions on Knowledge Discovery from Data (TKDD), 1(1): 2.

Leydesdorff, L. (2001). "Technology and Culture: The Dissemination and the Potential Lock-in of New Technologies", Journal of Artificial Societies and Social Simulation, 4(3), http://jasss.soc.surrey.ac.uk/4/3/5.html.

Liljeros, F., C. Edling, L. Amaral, H. Stanley, and Y. Aaberg. 2001. "The Web of Human Sexual Contacts". Nature 411(6840): 907-908.

McCulloh, I., J. Lospinoso, and K.M. Carley. 2010. "The Link Probability Model: A Network Simulation Alternative to the Exponential Random Graph Model", Internal report (No. CMU-ISR-10-130), Carnegie-Mellon University Pittsburgh, Center for Computational Analysis of Social and Organizational Systems.

Menezes, T., and C. Roth 2014. "Symbolic Regression of Generative Network Models" Scientific reports, 4.

Moreno, S., S. Kirshner, J. Neville, and S.V.M. Vishwanathan. 2010. "Tied Kronecker Product Graph Models to Capture Variance in Network Populations", In Proceedings of the 48th IEEE Annual Allerton Conference on Communication, Control, and Computing (Allerton), 2010: 1137-1144.

Robins, G., P. Pattison, Y. Kalish, and D. Lusher. 2007. "An Introduction to Exponential Random Graph (p*) Models for Social Networks", Social Networks 29(2): 173-191.

Robins, G.L., T.A.B. Snijders, P. Wang, M. Handcock, and P. Pattison. 2007. "Recent Developments in Exponential Random Graph (p*) Models for Social Networks”, Social Networks 29:192-215.

Snijders, T.A., P.E. Pattison, G.L. Robins, and M. Handcock. 2006. "New Specifications for Exponential Random Graph Models", Sociological Methodology 36:99-153.

Soorapanth, S., S.E. Chick, and J.S. Koopman. 2001. "Simulation of Stochastic Infection Transmission Models Designed to Inform Water Treatment Decisions." In Proceedings of the 13th European Simulation Symposium (Marseille, France, Oct. 18-20). SCS Publications, 517-521.

Stauffer, D., A. Sousa, and C. Schulze (2004). "Discretized Opinion Dynamics of the Deffuant Model on Scale-free Networks", Journal of Artificial Societies and Social Simulation, 7(3), http://jasss.soc.surrey.ac.uk/7/3/7.html.

Stocker, R., D.G. Green, and D. Newth. (2001). "Consensus and Cohesion in Simulated Social Networks", Journal of Artificial Societies and Social Simulation, 4(4), http://jasss.soc.surrey.ac.uk/4/4/5.html.

Suo, S., and Y. Chen (2008). "The Dynamics of Public Opinion in Complex Networks", Journal of Artificial Societies and Social Simulation, 11(4), http://jasss.soc.surrey.ac.uk/11/4/2.html.

Tesfatsion, L. 2002. "Agent-Based Computational Economics: Growing Economies from the Bottom Up”, Artificial Life, 8(1):55-82. 
Thiriot, S., and J.D. Kant. 2008. "Generate Country-Scale Networks of Interaction from Scattered Statistics". In The fifth conference of the European social simulation association, Brescia, Italy (Vol. 240).

Van Duijn, M., and T.A.B. Snijders. 1995. "The P2 Model” Internal publication, VSM, University of Groningen.

Watts, D.J., and S.H. Strogatz. 1998. "Collective Dynamics of Small-World Networks". Nature 393(6684): 440-442.

Watts, D.J. 1999. Small Worlds: The Dynamics of Networks between Order and Randomness. Princeton University Press, Princeton, New Jersey.

Wolfram, S. 1986. Theory and Applications of Cellular Automata. Singapore: World Scientific.

Yang, C., S. Kurahashi, K. Kurahashi, I. Ono, and T. Terano (2009). "Agent-Based Simulation on Women's Role in a Family Line on Civil Service Examination in Chinese History", Journal of Artificial Societies and Social Simulation, 12(2), http://jasss.soc.surrey.ac.uk/12/2/5.html.

\section{AUTHOR BIOGRAPHIES}

FREDERIC AMBLARD is Associate Professor of Computer Science at University Toulouse 1 Capitole and associated researcher at the Institut de Recherche en Informatique de Toulouse (IRIT, UMR CNRS 5505) in the SMAC (Cooperative Multi-Agent Systems) team. He holds M.S. degree from the Montpellier University and Ph.D. degrees in Computer Science from Blaise Pascal University. His works concern mostly agent-based social simulation of influence dynamics, opinion dynamics and social networks. His email address is frederic.amblard@ut-capitole.fr.

AUDREN BOUADJIO-BOULIC is a PhD student at University Toulouse 1 Capitole in the Institut de Recherche en Informatique de Toulouse (IRIT, UMR CNRS 5505) in the SMAC (Cooperative MultiAgent Systems) team. His e-mail address is audren.bouadjio@gmail.com.

CARLOS SUREDA GUTIERREZ is a PhD student at University Toulouse 1 Capitole in the Institut de Recherche en Informatique de Toulouse (IRIT, UMR CNRS 5505) in the SMAC (Cooperative MultiAgent Systems) team. His e-mail address is csureda@irit.fr.

BENOIT GAUDOU is Associate Professor of Computer Science at University Toulouse 1 Capitole and associated researcher to the Institut de Recherche en Informatique de Toulouse (IRIT, UMR CNRS 5505) in the SMAC (Cooperative Multi-Agent Systems) team. He holds M.S. and Ph.D. degrees in Computer Science from University Paul Sabatier. His main research interests concern cognitive agents modelling and simulation, he is strongly involved in the development of the GAMA simulation platform (https://code.google.com/p/gama-platform/). His e-mail address is benoit.gaudou@ut-capitole.fr. 